\title{
Erosões e cristalizações
}

\section{Erosions and crystallizations}

MICHAL KIRSCHBAUM

Universidade de Santa Catarina, UDESC, Florianópolis, Brasil

\section{RESUMO}

Este artigo versa sobre o trabalho de dois artistas visuais contemporâneos a partir de questões que se esperam relevantes para pensar a fotografia contemporânea. 0 texto coloca em reflexão dois trabalhos que problematizam a transparência da imagem e do olhar em relação a um referente. O texto parte da obra "Yucatan Mirror Displacements", de Robert Smithson (EUA, 1938-1973) afım de pensar questões relacionadas a visualidade e percepção sobre o tempo e o espaço e alonga as reflexões ao encontro do trabalho "Killed Negatives (After Walker Evans)", de Lisa Oppenheim (EUA, 1975). Ao longo do texto se propõem conversas com outros autores como Jacques Derrida, Yves-Lomax, Robert Smithson, Susan Sontag e Jeniffer Roberts.

\section{PALAVRAS-CHAVE}

Fotografia, operações espaciais, desconstrução.

\begin{abstract}
A B STR ACT
This article discusses the work of two contemporary visual artists based on questions that are expected to be relevant for thinking about contemporary photography. The text puts into reflection two works that problematize the transparency of the image and the look in relation to a referent. The text is based on the work "Yucatan Mirror Displacements", of Robert Smithson (USA, 1938-1973) in order to think about issues related to visuality and perception about time and space and extends the reflections to meet the work "Killed Negatives (After Walker Evans)", of Lisa Oppenheim (USA, 1975). Throughout the text, conversations are proposed with other authors such as Jacques Derrida, Yves-Lomax, Robert Smithson, Susan Sontag and Jeniffer Roberts.
\end{abstract}

\section{KEYWORDS}

Photography, spatial operations, deconstruction. 


\section{Erosões e cristalizações}

No ensaio fotográfico "Incidents of Mirror Travel in the Yucatan", publicado na Revista Artforum por Robert Smithson em 1969, as vozes de deuses maias revelam a percepção como algo turvo e instável. 0 espelho, material que Smithson usa para produzir as instalações que posteriormente fotografa, é o objeto pelo qual os deuses maias falam, como Tezcatlipoca, o deus do espelho de obsidiana. As instalações com espelhos e a interferência dos deuses dividem a realidade e indicam os cortes e as separações que já são existentes, entre a realidade e seus reflexos, entre a realidade e as percepções que podemos reconstruir desta. Jeniffer Roberts coloca que Smithson usa os espelhos como uma "ferramenta de corte ou separação em vez de uma espécie de ferramenta de unificação" (ROBERTS, 2004, p. 47) (tradução nossa). ${ }^{1}$ Aos poucos os reflexos e os deslocamentos ativam um fluxo fantasmagórico que intervém no presente como injunções e disjunções do cotidiano.

Comecei a pensar em trabalhos que são movidos a partir de algo que identifiquei como uma provocação. Parece que Smithson foi inicialmente provocado pelo travelogue de John Lloyd Stephens publicado em 1843 com o título "Incidents of Travel in Yucatán”. Porém o artista faz uma espécie de anti-expedição de Stephens.

A expedição de Stephens tinha um propósito que diferia radicalmente da expedição de Smithson considerando as operações imperialistas do travelogue de 1843 que ía ao encontro das ruínas maias da região. O livro de Stephens conta com imagens que se tornaram uma referência importante da arqueologia mesoamericana (ROBERTS, 2004). Smithson não critica Stephens diretamente mas ao longo de seu texto podem ler-se posições adversas, especialmente em suas menções a desconfiança do aparelho visual para representar o outro de forma realista. A partir do trabalho Enantiomorphic Chambers (1964) de Smithson fica clara a sua posição quanto a descrição realista, apontada como uma operação mental ilusionista e mostrando o poder da perspectiva central e da operação ilustrativa sobre o outro. Sua anti-expedição não busca dados objetivos e expõe os procedimentos da instalação da visão moderna para a "extração de artefatos históricos e seu deslocamento para os Estados Unidos, o novo epicentro histórico pan-americano" (ROBERTS, 2004, p. 94-96) (tradução nossa). ${ }^{2}$

Seu ensaio fotográfico se mostra também atravessado por provocações internas, conversas entre meios onde o texto se articula "fotograficamente", onde fotografias rompem as sequencialidades tempo-espaciais dos lugares e do texto. Essas conversas vão constantemente re/ materializando o espaço, os meios, as entidades através do texto do artista, abrindo lugar para um trânsito entre diferentes linguagens- ou o encontro de naturezas distintas, como se ilustrassem o próprio processo do pensamento. Uma coisa e depois uma outra: uma sequencia; E logo depois uma simultaneidade, um salto, uma volta, por fim uma série de decomposições, ou cristalizações: forças que insistem entre conservar e alterar as diversas formações.

1 No original: "a tool of cutting or splitting rather than strict unification" (ROBERTS, 2004, p. 47).

2 No original: “" the extraction of historical artifacts and their relocation to the United States, the new PanAmerican historical epicenter.” (ROBERTS, 2004, p. 94-96). 
A mente e a terra encontram-se em um processo constante de erosão: rios mentais derrubam encostas abstratas, ondas cerebrais desgastam rochedos de pensamento, ideias se decompõem em pedras de desconhecimento, e cristalizações conceituais desmoronam em resíduos arenosos de razão. Vastas faculdades móveis acontecem neste miasma geológico, e elas se movem da maneira mais física possível. Esse movimento parece ser sem movimento, ainda assim ele esmaga a paisagem da lógica sob devaneios glaciais. Esse fluir lento nos faz conscientes da turbidez do pensamento (SMITHSON, 1996, p. 100)(tradução nossa) ${ }^{3}$

A situação turva colocada em operação articula um espaço multi-temporal e multimidiático, pois desconstrói o espaço enquanto texto e enquanto fotografia e no transcorrer de uma narrativa que abre lugar à ficção os objetos tomam lugar de sujeitos, incorporam vozes ou, (vide " $A$ Tour in the Monuments of Passaic", 1967) se sexualizam.

As discussões dentro do contexto do Minimalismo podem esclarecer alguns pontos do trabalho de Smithson. De fato a dualidade sujeito-objeto é algo que o "minimalismo tenta superar na experiência fenomenológica” (FOSTER, 1996, p. 40)(tradução nossa), e traz dessa forma a teatralidade apontada por Michael Fried, que incorpora o tempo e o espaço à obra. Para Fried a teatralidade estava infectando a arte moderna, tirando a "significação própria" da obra de arte por apontar a sua externalidade (FRIED, 1998). O autor estava correto nesse sentido, em que, colocando o caráter de objeto à obra esta nos levaria para um olhar circunstancial colocando então um contexto em evidência. Tal posição desvelaria que muitos trabalhos desse contexto operariam no espaço expositivo levando um foco à dinâmica obra-participante, e aos agenciamentos em torno do projeto expositivo. Exatamente por um foco na fisicalidade do objeto, um deslocamento do objeto para a cena, da matéria para os fluxos que a atravessam. Através de uma literalidade do objeto artístico o aspecto de teatralidade na obra deixou claro que a obra se dá em relação à experiência, por onde o sentido da obra se estrutura.

3 No original: One's mind and earth are in a constant state of erosion, mental rivers wear away abstract banks, brane waves undermine cliffs of thought, ideas decompose into stones of unknowing and conceptual crystallizations break apart into deposits of gritty reason. Vast moving faculties occur in this geological mias$\mathrm{ma}$, and they move in the most physical way. This movement seems motionless, yet it crushes the landscape of logic under glacial reveries. This slow flowage makes one conscious of the turbidity of thinking. (SMITHSON, 1968, p. 100). 


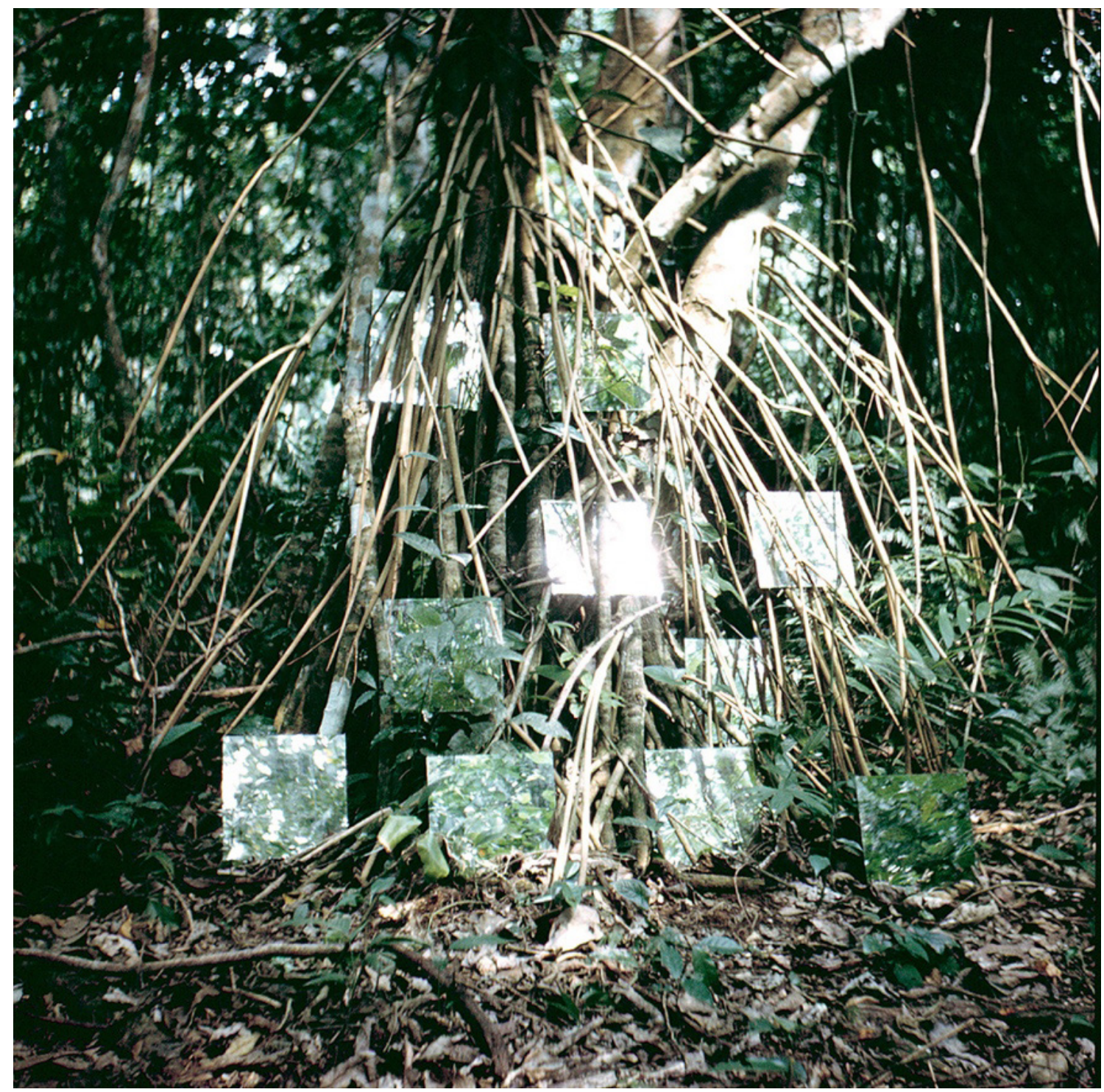

Figura 1 - Robert Smithson. Yucatan Mirror Displacements (1-9), 1969, Fotografia, 61 x $61 \mathrm{~cm}$ cada fotografia Coleçao Museu Guggenheim. Fonte: https://www.guggenheim.org/artwork/5322.

A percepção do tempo e do contexto ao vivenciar um trabalho de arte rompe qualquer possibilidade deste trabalhar por revelações de um presente perpetuo. 0 trabalho pode então, mesmo que "literal", articular uma perspectiva transcendental, considerando a transformação: as coisas, de fato, estão sempre em eterna mudança: se apresentam em um sem fim de camadas de tempo pelas sedimentações entrópicas visíveis, invisíveis e projetadas.

Esse é um dos pontos que Incidents of Mirror Travel in the Yucatan (figura 1) reivindica. A transcendência tem uma perspectiva entrópica, e este é um motor poético de importância no trabalho de Smithson, que leva em conta os processos de violência (na perspectiva geológica e histórica) que podem ser percebidos ao lermos o presente. Se um presente dado está relacionado 
a eventos catastróficos e traumáticos do passado como poderia este se mostrar sem interrupções? Como seria possível ler a realidade de forma uniforme e não plural?

Os significados e entendimentos sobre algo são necessariamente articulados por reconstruções e estão sempre abertos a novos sentidos que possam vir a acontecer. Outra perspectiva, um tanto mais abstrata, propõe que algo mais poderia estar acionando os entendimentos, percepções e sentidos das coisas. Essa perspectiva aponta a que estes também são produzidos por algo que nos atravessa e que incorporamos a partir de vozes que intervém, como injunções, em nosso tempo. Percebem-se aqui como vozes que não são exatamente nossas mas que falam através de nós, algo que talvez possa ser entendido dentro dos discursos, dos mandatos, das injunções somando-se a uma perspectiva fantasmagórica por onde outros falam através de mim.

Esse olhar vem a incrementar a existência multifacetada e multitemporal de qualquer tempo e lugar, apontando às muitas possibilidades que desestabilizam qualquer realidade. O objetivo de definir algo, pelo bem de uma articulação, no sentido do definitivo, começa a ser um caminho sem sentido na configuração atual. Como pode o conhecimento ser algo se for sem uma destinação pontual? Um olhar nas "coisas em movimento" pode nos surpreender com uma compreensão escorregadia:

É preciso significar, é preciso filtrar, peneirar, criticar, é preciso resolver os vários possíveis que habitam a mesma injunção. E habitá-la de uma forma contraditória em meio a um segredo. Se a legibilidade de um legado fosse dada natural, transparente, unívoca, se não exigisse e ao mesmo tempo desafiasse sua própria interpretação, nunca teríamos nada a herdar. (DERRIDA, 1994, p. 16) (tradução nossa). ${ }^{4}$

Os vários possíveis que estão presentes na mesma existência desafiam a manifestação de qualquer presença por inteiro. De uma dada existência que nos assalta, a partir de qual somos chamados a habitá-la, ela é inteira somente na sua possível percepção promíscua, inteira na sua essência de ser feita de vários e de nunca ser completa ou concluída pois está sempre pronta a interromper e ser interrompida.

As interrupções e desconstruções colocadas em prática por Robert Smithson são ações que são impulsionadas por suas considerações em relação ao enantiomorfismo e sua relação com o sistema visual como um sistema de desconstrução-reconstrução. O enantiomorfismo aponta a uma quebra de unidade em relação a natureza descontínua da experiência e do sentido e deixa em evidência que os processos de leitura ou recepção são uma construção interferidos por nosso enantiomorfismo interno cortando a duração em pedaços: uma temporalidade desconexa implícita em nosso olhar.

4 No original: One must means one must filter, sift, criticize, one must sort out several different possibles that inhabit the same injunction. And inhabit it in a contradictory fashion around a secret. If the readability of a legacy were given, natural, transparent, univocal, if it did not call for and at the same time defy interpretation, we would never have anything to inherit from it. (DERRIDA, 1994, p. 16) 
Que o enantiomorfismo oferece uma aproximação aceitável da différance (a palavrachave de Derrida para a diferença que escapa, precede e constitui toda presença) - uma diferença que opera espacial e temporalmente, que difere e adia. (ROBERTS, 2004, p. 49) (tradução nossa) ${ }^{5}$

Uma presença, dito que se forma através de um processo construtivo, nunca se mostra inteiramente, como coloca Derrida, já que seu sentido e presença são plurais e nunca inteiramente manifestado, diferindo e adiando seu sentido. Différance parece estabelecer a desconstrução 6 que qualquer presença é sempre sujeita, diferindo e adiando seu entendimento dentro de sua própria arquitetura e em tempo, novamente habitada, alterando-a mas também trazendo-a de volta, de maneira singular, pois específica, pela "teatralidade" que circunscreve qualquer existência. Como coloca Derrida: "Não há différance sem alteridade, não há alteridade sem singularidade, não há singularidade sem o aqui e agora." (DERRIDA, 1994, p. 31) (tradução nossa)7

Husserl explicou o "presente" como um ponto que sempre divergia para a frente em protensão e para trás em retenção. Smithson possuía uma cópia de The Phenomenology - sua influência é clara quando ele afirma, por exemplo, que em um de seus projetos de viagem "o presente caiu para frente e para trás em um tumulto de 'desdiferenciação'. Em cada tentativa de entendermos o presente, encontraremos apenas um vazio, um espaço suspenso pelas ligaduras reflexivas de protensão e retenção." (ROBERTS, 2004, p. 50) (tradução nossa). ${ }^{8}$

5 No Original: That enantiomorphism offers a passable approximation of différance (Derrida's keyword for the difference that evades, precedes, and constitutes all presence- a difference which operates both spatially and temporally, which both differs and defers. (ROBERTS, 2004, p. 49).

6 Em outro lugar, Derrida expande essa imbricação do trabalho de desconstrução e memória quando explica que "a própria condição de uma desconstrução pode estar em trabalho, no trabalho, dentro do sistema a ser desconstruído; já pode estar localizado ali, já em trabalho "..." a desconstrução não é uma operação que acontece depois, desde fora, em um belo dia; ela já está sempre em ação no trabalho ... Como a força disruptiva da desconstrução já está sempre contida na arquitetura do trabalho, tudo o que seria necessário finalmente fazer para poder desconstruir, dado esse sempre já estar ali, é fazer um trabalho de memória”. (RICHTER, 2010, p. 62) (tradução nossa).

No original: Derrida elsewhere expands on this imbrication of deconstruction and memory work when he explains that "the very condition of a deconstruction may be at work, in the work, within the system to be deconstructed; it may already be located there, already at work"..."deconstruction is not an operation that supervenes afterwards, from the outside, one fine day; it is always already at work in the work.... Since the disruptive force of deconstruction is always already contained within the architecture of the work, all one would finally have to do to be able to deconstruct, given this always already, is to do memory work" (RICHTER, 2010, p. 62)

7 No original: "No differance without alterity, no alterity without singularity, no singularity without here-now." (DERRIDA, 1994, p. 31).

8 No original: "Husserl explained the "present" as a point that was always diverging forward into protention and backward into retention. Smithson owned a copy of The Phenomenology - its influence is clear when he claims, for example, that in one of his traveling projects "the present fell forward and backward into a tumult of 'de-differentiation" "In each attempt to grasp at the present, one will encounter only a void, a gap suspended by the reflective ligatures of protention and retention." (ROBERTS, 2004, p. 50). 
O que ocorre então se parte de uma imagem de retenção, como uma fotografia, como um instante em repetição infinita, tem o seu eco interrompido? E então aquele momento que damos por estabilizado imageticamente é interferido, parcialmente destruído. Uma lacuna, praticada em um meio que é o da representação, estabelece então um significado que parte de uma performatividade que toma lugar sobre esta. O não visível vai se tornando visível pois a produção de ausências é uma forma de interromper o que é visível. Um trabalho de Lisa Oppenheim me provocou imensamente a pensar as questões acima delineadas no qual me aprofundarei na sequência.

Lisa Oppenheim (EUA, 1975) trabalha nos ecos que reverberam da relação entre o visível e o não-visível, apresentando-se como um lugar potente de exploração no campo da fotografia. Suas propostas as vezes tornam mais complexo o entendimento da fotografias como um processo transparente.

Os espaços de representação, na pluralidade de suas próprias possibilidades, "pode ser encenada somente em múltiplas vozes", habitada em múltiplas vozes, como a chamada de um outro, "e sua encenação futura somente pode ser escrita e pensada em múltiplas vozes, ainda que alusivas e espectrais." (RICHTER, 2010, p. XXXVII) (tradução nossa) ${ }^{9}$. Ao mesmo tempo, Derrida percebe certos meios, referindo-se à fotografia e ao filme, com uma natureza espectral 10 pela sua potência que intercepta o presente, como um eco que cria multiplicidades temporais no presente.

A câmera torna a realidade atômica, gerenciável e opaca. É uma visão de mundo que nega interconexão, continuidade, mas que confere em cada momento o caráter de um mistério. Qualquer fotografia tem múltiplos significados, de fato, ver algo na forma de uma fotografia é encontrar um objeto potencial de fascínio. A sabedoria suprema da imagem fotográfica é dizer: "Existe uma superfície - agora pense - ou melhor sinta, intua - o que está além dela, como a realidade deve ser se ela for assim." Fotografias que não podem explicar nada são convites inesgotáveis para a dedução, a especulação e a fantasia. (SONTAG, 1978, p.22-23). 11

9 No original: "can only be staged in multiple voices"..."and their future staging can only be written and thought about in multiple voices, ones that remain elusive and spectral." (RICHTER, 2010, p. XXXVII).

10 "E eu acredito que desenvolvimentos modernos na tecnologia e nas telecomunicações em vez de diminuir as fantasmagorias assim como qualquer pensamento científico ou técnico abandona a era dos fantasmas, como parte de uma era feudal com suas tecnologias primitivas, como se fosse uma era perinatal...eu acredito que fantasmas fazem parte do futuro e que a tecnologia moderna das imagens como o cinema e as telecomunicações intensificou o poder dos fantasmas e sua habilidade de assombração". (Transcrição e tradução nossa do discurso de Derrida em Ghost Dance, 1983). No original: "And I believe that modern developments in technology and telecommunication instead of diminishing the realm of ghosts as does any scientific or technical thought is leaving behind the age of ghosts, as part of the feudal age with its somewhat primitive technology as a certain perinatal age. Whereas I believe that ghosts are part of the future. And that the modern technology of images like cinematography and telecommunication enhances the power of ghosts and their ability to haunt us (Transcript of Derrida's speech in Ghost Dance, 1983) (MCMULLEN, 1984)

11 No original: The camera makes reality atomic, manageable, and opaque. It is a view of the world which denies interconnectedness, continuity, but which confers on each moment the character of a mystery. Any photograph has multiple meanings, indeed, to see something in the form of a photograph is to encounter a potential object of fascination. The ultimate wisdom of the photographic image is to say: "There is the surface 

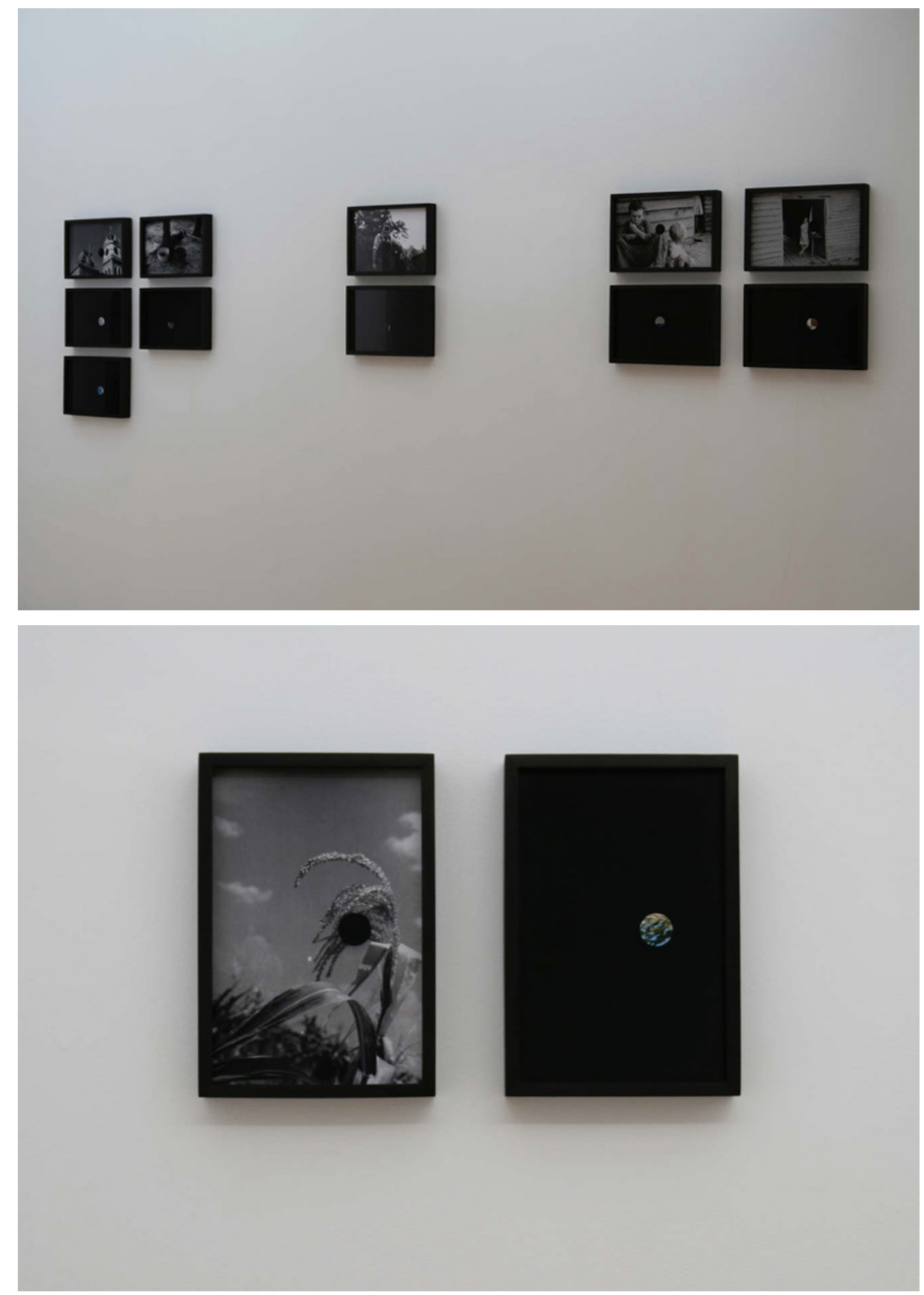

Figura 2 - Lisa Oppenheim. Killed Negatives (after Walker Evans), 2007, Fotografia, 20 x $30 \mathrm{~cm}$ cada fotografia. Fonte: https://lisaopp.net/ij-2008.

- now think - or rather feel, intuit - what is beyond it, what the reality must be like if it looks this way." Photographs which cannot themselves explain anything, are inexhaustible invitations to deduction, speculation, and fantasy. (SONTAG, 1978, p. 22-23) 
A fotografia traz um eco a ser habitado. A referência, distante e inalcançável, volta de uma origem que não pode ser alcançada mas repetida em novas situações e contextos.

Em “Killed Negatives (after Walher Evans)" (figura 2) as operações feitas sobre um arquivo fotográfico são colocadas em evidência trazendo a história da fotografia como lugar potente para pensar diversas questões. ${ }^{12}$ Essas questões se percebem através de um vestígio evidente, não somente em relação a esta escrita de luz a que chamamos fotografia, mas a um recorte feito no material fotográfico.

Tive a chance de assistir aos vídeos que faziam parte da inscrição de Lisa Oppenheim para a Rijksacademy em Amsterdam, em um deles, intitulado "Palmspring California" consta um texto escrito pela artista e insertado na caixa do disco compacto, onde se pode ler:

Eu vim até este ponto e não sabia ao certo onde colocar a câmera. Se eu a posicionasse do outro lado da estrada como antes eu pretendia, uma alta cerca limitando o campo de golfe, onde o Bob Hope Invitational é realizado todos os anos, teria dominado a imagem. Com quanto cuidado as equipes de televisão que cobrem o evento devem apontar suas câmeras para não permitir que a audiência veja a interminável faixa de areia do outro lado da cerca. (OPPENHEIM, 2004) (tradução nossa).

O interesse de Oppenheim na representação de lugares e na produção do olhar se faz evidente. Em outro material audiovisual intitulado "Palmdale, California" é também evidente seu foco em como o desenvolvimento e as escavadeiras estavam modificando o lugar e o meio ambiente.

A sua colocação sobre como a imagem é produzida e cuidadosamente enquadrada para distribuição em relação ao que é dado a ver também deixa claro seu interesse nas articulações que estão por trás da produção visual e sua posterior manipulação nos processos de distribuição, exibição e arquivamento. Os trabalhos então levantam questionamentos sobre como os lugares são produzidos por conceitos, sobre como conceitos são produzidos pela tecnologia, e sobre como lugares e percepções (compartilhados coletivamente) são produzidos pela tecnologia. Essas questões também vem a perguntar como todas essas instâncias interferem na percepção sobre os lugares, produzindo parte de suas significações e modos de experimentá-los.

Se uma tecnologia, um método específico de produção, faz perceptível um modo específico de operação, pode-se dizer que o mesmo pode apresentar dentro de seu próprio sistema, um modo de operar sobre/com o mundo. Rattemeyer aponta que alguns de seus trabalhos foram produzidos

12 Christian Rattemeyer comenta sobre o trabalho da artista: "A prática de Oppenheim esta sempre em sintonia fina com as possibilidades de significado que são reveladas quando a história técnica do meio é lida contra si mesma, abrindo fissuras entre o formal e o tecnológico"... "ela re-fotografou o buraco em "killed negatives" de Walker Evans. Nesses trabalhos os meios formais e seus efeitos se tornam ambos objeto e sujeito de seus sentidos" (RATTEMEYER, 2010) (traducão nossa). No original: "Oppenheim's practice is always finely attuned to the possibilities of meaning that are revealed when the technical history of the medium is read against itself, opening fissures between the formal and the technological."..."she has re-photographed the missing punch dot in "killed negatives" by Walker Evans. In those works"..."the formal means and their effects become both object and subject of their meaning." (RATTEMEYER, 2010) 
em relação ao seu interesse nas "intersecções entre o tecnológico e o conceitual na lógica de representação da Fotografia"13 (RATTEMEYER, 2010) (tradução nossa). Ainda que seu interesse se localiza na problemática da produção da imagem este também parece tocar a problemática de como somos afetados por essas imagens.

Em “Killed Negatives: After Walker Evans”, Lisa Oppenheim trabalha sobre as fotografias não publicadas de Walker Evans de 1938. A historia da produção desse material é a de que Walker Evans foi contratado pela "Farm Security Administration" para documentar a Depressão na Norte América rural. Essas imagens se tornaram um material importante para a história visual da Depressão norte americana. Parte desse legado, arquivado na Biblioteca Nacional do Congresso, sofreram recortes como forma de impedir a publicação de parte desse material. Os negativos que Oppenheim selecionou para seu trabalho são aquelas que foram censurados durante a operação administrativa sobre esse material. 0 diretor do programa para documentação da sociedade norte americana da época, Roy Emerson Stryker, editou alguns dos negativos e rejeitou alguns perfurando-os para que não fossem publicados e deu-lhes o nome de "killed negatives": negativos mortos. A partir desse material "não-oficial", foi possível colocar em evidência este ato de censura, e ver as operações que estão presentes nos processos de arquivamento, muitas vezes invisíveis. Utilizando estes materiais, Lisa Oppenheim coloca essas perfurações como a característica central dessas imagens, como um espaço de potencialidades contemporâneas.

A perfuração do negativo, materializada em um ponto preto em sua ampliação deixou evidente a materialização de uma ausência que parece ao mesmo tempo acionar e destruir a memória pela autoridade documental de uma época. Tal contexto aponta de certa forma para uma tensão entre o histórico e uma materialidade, e para a historia também como uma acumulação de material, através de seleções, abandonos e descartes.

É interessante ver, que nesse processo, o material original, no caso o negativo, se torna presente, enquanto que normalmente é algo desconsiderado. A ampliação do negativo, espelha o ato da censura, e rompe a transparência do negativo por ter parte ausente. Finalmente a origem da fotografia e seu ato de censura ecoam juntos para serem lidos, preenchidos e habitados. Há um ponto que falta, uma parte que falta e que trabalha como estímulo a algo que tem como essência mesmo a falta: o desejo de preenche-lo.

A obsessão da artista sobre este ponto faltante a leva a uma busca para capturar a visibilidade que não está no negativo. Ela fotografa então locais próximos a fim de trazer de volta esse ponto ausente. No entanto, ela não busca fazer uma costura ou preencher o buraco. A artista faz uma outra fotografía onde o buraco se faz visível com uma imagem e a sua volta o papel se mostra completamente preto, com a mesmo obscuro que antes se percebia na perfuração. Novas áreas indecifráveis estão localizadas a partir de uma ação que nos dá a sensação de estar olhando através do buraco de uma fechadura onde a obsessão fica localizada somente na parte faltante, no recorte. É pertinente pensar essa questão em relação a linguagem fotográfica, que cria espectros e

13 No original: "intersections between the technological and the conceptual in the representational logic of photography” (RATTEMEYER, 2010). 
imagens de culto e que em si também é um recorte de um referente inalcançável.

Os pontos fotografados por Oppenheim são captados em cor, algo que coloca em evidência a distância dessas das fotografias preto e branco de Walker Evans. O tempo é colocado em evidência e a percepção de uma completude inalcançável é estressada, fornecendo-nos uma dupla perspectiva. A dupla perspectiva temporal no trabalho de Oppenheim cria um sentimento de estranheza em relação ao presente, especialmente ao considerar que estamos diante do meio fotográfico, tecnologia que coloca em evidência a passagem do tempo. A tensão se instala entre o referente e sua desintegração, entre uma totalidade parcial e um fragmento. Apesar que o negativo já tinha sido perfurado pelo diretor do programa de documentação do mencionado arquivo, o furo deixa mais evidente a realidade inalcançável.

Enquanto eu corto, é revelado que por trás de uma imagem existe apenas outra imagem. Uma representação refere-se a outra"..."Conforme eu corto é revelado que não há verdade literal adequada que tenha sido mascarada pela imagem. Atrás da superfície fotográfica não há uma realidade segura e completa”..."Atrás da fotografia não há um objeto real, somente outra imagem"14 (LOMAX, 1985, p. 266-267) (tradução nossa).

Um encontro com visualidades interrompidas é acionado por Robert Smithson e Lisa Oppenheim, ora questionando o olhar e as formas de leitura e colocando em evidência múltiplas perspectiva ora no caso de Oppenheim também atentando às ações que estão no contexto de criação e distribuição das imagens. Os procedimentos também investem em um olhar para a experiência de ser um observador desses lugares e objetos de onde surge um senso misto de expectativa, uma forma de ansiedade entre as imagens e o referente não encontrado.

Os trabalhos também colocam em evidência a fotografia em um campo expandido, como uma prática que toma lugar dentro de uma cadeia de processos de circulação, articulação e idealizações por onde a fotografia "presta testemunho na medida em que ativa a circulação de uma certa memória cultural e produz intercâmbios através de seus modos de escrita, inspeção e interpretação específicos do meio" 15(RICHTER, 2010, p. XX5) (tradução nossa).

14 No original: "As I cut, it is revealed that behind one image there is but another image. One representation refers to another"..."As I cut it is revealed that there is no proper literal truth which has been masked by the image's front. Behind the photographic surface there isn't a sure and whole reality"..."Behind the photograph there is no real object, only another image". (LOMAX, 1985, p.266-267).

15 No original: "bears witness in that it activates the circulation of a certain cultural memory and exchange through its medium-specific modes of writing, inspection, and interpretation." (RICHTER, 2010, p. XXV). 


\section{Referências}

DERRIDA, J. Injunctions of Marx. IN: Specters of Marx: the state of debt, the work of mourning, and the New International. Nova lorque: Routledge, 1994.

FLAM, J. (ed) Robert Smithson: The Collected Writings. Londres: University of California, 1996.

FRIED, M. Art and Objecthood. Chicago: The University of Chicago Press, 1998.

FOSTER, $\mathrm{H}$. The Return of the Real: the avant-garde at the end of the century. Estados Unidos: MIT Press, 1996.

HOLT, N. (ed). The Writings of Robert Smithson. Essays with illustration. New York Nova lorque: University Press, 1979.

LOMAX, Y. 'Re-Vision'. IN: Re-visions: fringe interference in British Photography in the 1980s. Cambridge: Cambridge Darkroom, 1985.

MCMULLEN, K.(dir/prod) Ghost Dance. EUA: produção independente, 1984. (100 min): mono., cor, $35 \mathrm{~mm}$.

OPPENHEIM, L. A Free Ride. Inscrição de Lisa Oppenheim a residência da Rijksacademy. Biblioteca da Rijksacademy. DVD. 10'10", 2004

OPPENHEIM, L. Dioptric, Oasis, Lili on Air. Inscrição de Lisa Oppenheim a residência da Rijksacademy. Biblioteca da Rijksacademy. DVD. 7'18", 2004

RATTEMEYER, C. Lunagrams: Lisa Oppenheim. Fantom Photographic Quarterly. Vol. 3, Primavera de 2010. p. 6-9). Fonte: disponível em http://www.fantomeditions.com/fantom-03-spring-2010. Acesso: 13/05/2020

ROBERTS, J. Mirror-travels: Robert Smithson and History. Londres: Yale Universtity Press, 2004.

SONTAG, S. On Photography. Estados Unidos: Farrar, Straus and Giroux, 1978. 


\section{Sobre o(a) autor(a)}

É artista, pesquisadora e docente. Doutoranda do programa de pós-graduação em Artes Visuais da Universidade do Estado de Santa Catarina (UDESC) e mestre em Investigação Artística pela Universidade de Amsterdam (UvA).

Orcid: https://orcid.org/0000-0002-6270-0761

Recebido em: 15-05-2020 / Aprovado em: 30-06-2020

\section{Como Citar}

Kirschbaum, Michal (2020). Erosões e cristalizações. Revista Estado da Arte, Uberlândia. v.1, n.1, p. 19-31, jan./jun. 2020. https://doi.org/10.14393/EdA-v1-n1-2020-54802 